OPEN ACCESS

Edited by:

Fuat Balcl,

Koç University, Turkey

Reviewed by:

Sander Andries Los,

Vrije Universiteit Amsterdam

Netherlands

Giovanna Mioni,

University of Padua, Italy

*Correspondence:

Marcus Missal

marcus.missal@uclouvain.be

Specialty section:

This article was submitted to Learning and Memory,

a section of the journal

Frontiers in Behavioral Neuroscience

Received: 25 July 2019 Accepted: 31 October 2019 Published: 22 November 2019

Citation:

Hsu T-Y, Lee H-C, Lane TJ and Missal M (2019) Temporal Preparation, Impulsivity and Short-Term Memory in Depression.

Front. Behav. Neurosci. 13:258. doi: 10.3389/fnbeh.2019.00258

\section{Temporal Preparation, Impulsivity and Short-Term Memory in Depression}

\author{
Tzu-Yu Hsu1,2, Hsin-Chien Lee ${ }^{3,4,5}$, Timothy Joseph Lane ${ }^{1,2,6}$ and Marcus Missal/6,7* \\ ' Graduate Institute of Mind, Brain and Consciousness, College of Humanities and Social Sciences, Taipei Medical University, \\ Taipei, Taiwan, ${ }^{2}$ Brain and Consciousness Research Center, Taipei Medical University-Shuang Ho Hospital, New Taipei City, \\ Taiwan, ${ }^{3}$ Department of Psychiatry, School of Medicine, College of Medicine, Taipei Medical University, Taipei, Taiwan, \\ ${ }^{4}$ Department of Psychiatry, Taipei Medical University Hospital, New Taipei City, Taiwan, ${ }^{5}$ Research Center of Sleep Medicine, \\ College of Medicine, Taipei Medical University, Taipei, Taiwan, ${ }^{6}$ Graduate Institute of Medical Humanities, College \\ of Humanities and Social Sciences, Taipei Medical University, Taipei, Taiwan, ${ }^{7}$ Division of System and Cognition, Institute \\ of Neurosciences (IONS), Université catholique de Louvain (UCLouvain), Brussels, Belgium
}

Patient suffering of major depressive disorder (MDD) often complain that subjective time seems to "drag" with respect to physical time. This may point toward a generalized dysfunction of temporal processing in MDD. In the present study, we investigated temporal preparation in MDD. "Temporal preparation" refers to an increased readiness to act before an expected event; consequently, reaction time should be reduced. MDD patients and age-matched controls were required to make a saccadic eye movement between a central and an eccentric visual target after a variable duration preparatory period. We found that MDD patients produced a larger number of premature saccades, saccades initiated prior to the appearance of the expected stimulus. These saccades were not temporally controlled; instead, they seemed to reflect reduced inhibitory control causing oculomotor impulsivity. In contrast, the latency of visually guided saccades was strongly influenced by temporal preparation in controls; significantly less so, in MDD patients. This observed reduced temporal preparation in MDD was associated with a faster decay of short-term temporal memory. Moreover, in patients producing a lot of premature responses, temporal preparation to early imperative stimuli was increased. In conclusion, reduced temporal preparation and short-term temporal memory in the oculomotor domain supports the hypothesis that temporal processing was altered in MDD patients. Moreover, oculomotor impulsivity interacted with temporal preparation. These observed deficits could reflect other underlying aspects of abnormal time experience in MDD.

Keywords: memory, depression, temporal cognition, eye movement, impulsivity

\section{INTRODUCTION}

Major depressive disorder (MDD) is often associated with an altered awareness of the passage time. Indeed, MDD patients often complain that subjective time is going by at a reduced pace compared with physical time (Gallagher, 2012; Msetfi et al., 2012; Ratcliffe, 2012; Droit-Volet, 2013; Thönes and Oberfeld, 2015; Davalos et al., 2018; Vogel et al., 2018). This perturbed time awareness has led 
to a systematic investigation of time "perception" using quantitative methods requiring an explicit judgment about durations. An explicit judgment about duration is the outcome of experimental tasks requiring comparison of time intervals, production or reproduction of a standard duration or verbal estimation (see Vatakis et al., 2018). This approach has led to conflicting results and the precise influence of depression on time perception remains elusive (see review in Oberfeld et al., 2014). However, temporal cognition is not limited to explicit temporal judgments. Implicit timing refers to the capacity to time actions based on temporal regularities in the environment (Coull and Nobre, 2008; Coull and Droit-Volet, 2018). It emerges in non-temporal tasks where temporal information is, nevertheless, essential to achieve optimal performance, as when making a saccade to a visual target. This implicit influence of elapsed time on movement preparation is often referred to as "temporal preparation" and is still poorly understood in depression (see Bonin-Guillaume et al., 2004).

Temporal preparation is studied, classically, by using a warning stimulus $\left(S_{1}\right)$ that predicts the occurrence of an imperative stimulus $\left(S_{2} ;\right.$ Woodrow, 1914). The period between $S_{1}$ offset and $\mathrm{S}_{2}$ onset is referred to as the foreperiod ("FP"; Niemi, 1981; Niemi and Näätänen, 1981). Temporal preparation buildsup while waiting during the FP and causes a shorter reaction time after $S_{2}$ appearance. Foreperiod duration could either remain constant, making the timing of $S_{2}$ entirely predictable, or FP duration could vary randomly between different values drawn from a given probability distribution. If FP duration is randomly drawn from a uniform probability distribution, the latency of the motor response to $S_{2}$ decreases with elapsed time. This "foreperiod effect" is the behavioral measure of temporal preparation. Temporal preparation could be explained by hypothesizing that subjects estimate the hazard rate of the target defined as the probability that $S_{2}$ will occur given that it has not occurred yet. As time elapses during the FP, the hazard rate of the $S_{2}$ increases and sensorimotor systems could use that information to reduce reaction time (Trillenberg et al., 2000; Janssen and Shadlen, 2005; Nobre et al., 2007). In addition, temporal preparation could also be modulated by the previous FP experienced by the subject (Alegria and Delhaye-Rembaux, 1975; Los and Van den Heuvel, 2001; Los et al., 2014, 2017). For instance, reaction time to $S_{2}$ appearance during the current FP will tend to be shorter, if the previous FP was shorter. Therefore, short-term temporal memory (i.e., sequence effects) plays a crucial role in temporal preparation (Los et al., 2017). Accordingly, it has been shown that the FP effect on saccadic eye movements could be accounted for by the remaining trace of previous FP duration (Ameqrane et al., 2014). This influence of short-term memory on the RT-FP function could be altered given the known impact of depression on memory (Burt et al., 1995). Therefore, altered temporal cognition in depression could be mainly due to a deficit of temporal memory.

Another factor that deeply influences response preparation in general is inhibitory control (Greenhouse et al., 2015; Lebon et al., 2016; Duque et al., 2017). More precisely, in order to prevent premature responses (i.e., responses before the onset of $\mathrm{S}_{2}$ ), inhibition is necessary to reduce the increasing tendency to initiate a motor response as time elapses (Burle et al., 2010; Correa et al., 2010). Inhibitory control is not only important for current FP, but also associated with short-term temporal memory. It has been suggested that when the preceding FP is longer than the current FP, inhibition induced during the preceding FP could cause a longer RT during the current FP. This indicates that inhibition could modulate the influence of short-term memory during the FP (Los, 2013). Therefore, it is plausible that the FP effect could be altered because of a dysfunctional inhibitory control. Inhibitory dysfunction is one aspect of impulsivity (Evenden, 1999). Impulsivity could be defined as the tendency to act without forethought and is commonly considered as one aspect of personality trait. To evaluate the magnitude of impulsivity, the Barratt Impulsiveness Scale (BIS-11; Patton et al., 1995) is often adopted. Studies have shown that impulsivity was exacerbated in neurologic (e.g., Parkinson's disease, Voon et al., 2011; Nombela et al., 2014), psychiatric (e.g., ADHD, Barkley et al., 2001; Smith et al., 2002) and affective disorders (e.g., MDD; see review in Evenden, 1999; Dalley and Robbins, 2017). There is a strong association between depression and impulsivity, suggesting that impulsivity could potentially lead to an increased risk of suicide (Corruble et al., 2003). Therefore, increased impulsivity in depression could cause more premature motor responses, altered temporal preparation and a different RT-FP function.

The aim of the present study was two-folds. Firstly, we examined whether temporal processing was altered in depressive patients by using a saccadic version of temporal preparation. Precise and accurate control of the timing of eye movements is essential to "catch" with the fovea the image of visual objects (Badler and Heinen, 2006; de Hemptinne et al., 2007; Barnes, 2008; Collins and Barnes, 2009). The same scenario also applies to temporal preparation. When both $S_{1}$ and $S_{2}$ are visual targets, temporal preparation plays a major role in oculomotor control (Oswal et al., 2007; Ameqrane et al., 2014; Degos et al., 2018). The saccadic system is kept under constant top-down inhibitory control in order to avoid unwanted eye movements that could blur the image of a visual object of interest on the retina during fixation periods (Rucker et al., 2011). The saccadic system therefore could be particularly sensitive to a lack of inhibitory control during temporal preparation and premature saccades and could be a valuable indicator of impulsivity. This phenomenon will be referred to as "oculomotor impulsivity" in this study. Additionally, according to the literature from above, short-term temporal memory and inhibitory control could potentially be altered and disrupt the FP effect in depressive patients. Thus, secondly, we further examined whether short-term temporal memory and inhibitory control affects temporal preparation in participants diagnosed with depression.

\section{MATERIALS AND METHODS}

\section{Ethical Approval and Informed Consent}

This study was approved by the Joint Institutional Review Board, Taipei Medical University, Taipei City, Taiwan (N201603080). Methods were carried out in accordance with relevant guidelines 
and regulations. All participants were informed about the purpose of the study and procedures before being asked to give informed consent. Written informed consent was obtained from all participants prior to their participation in this study.

\section{Patients}

Twenty-nine patients diagnosed with MDD (24 females; $38.4 \pm 2.5$ years old, $n=29$ ) were recruited by the Department of Psychiatry at Taipei Medical University Shuang-Ho Hospital, located in New Taipei City, Taiwan. The MINI-international neuropsychiatric interview (Sheehan et al., 1998), was used to confirm the diagnosis of current major depressive episode, to detect suicidal risk, and to exclude patients with psychotic symptoms and any comorbid mental disorder or substance use disorder according to the Diagnostic and Statistical Manual of Mental Disorders, Fifth Edition. Patients with poor visual acuity and comorbid medical conditions including neurological disorders (e.g., stroke, seizure, traumatic brain injury, post-brain surgery), brain implants (neurostimulators), cardiac pacemakers, or pregnant were also excluded. The Beck Depression Inventory (BDI-II; Beck et al., 1996), and the Generalized Anxiety Disorder 7 (GAD-7; Spitzer et al., 2006) were administered to evaluate the severity of depression and anxiety. BDI-II score for patients in this study was $30.1 \pm 13.4$ and depressive symptoms duration was $9.2 \pm 10.2$ years. In addition, the BIS-11 (Patton et al., 1995) was used to quantify Impulsive level on each individual. All patients but seven were on medication at the time of testing (see Table 1).

\section{Controls}

Twenty-nine healthy control participants (27 females, $37.7 \pm 2.4$ years old) without any current or history of neurological or psychiatric disorder, or use of psychotropic medication, were recruited from the community. They were matched for age and gender, except for two healthy control participants, whose gender did not match the patients.

\section{Experimental Design and Statistical Analysis}

Subjects were facing an LCD screen which presented stimuli at a refresh rate of $60 \mathrm{~Hz}$. An EyeLink 1000 infrared eye tracking system (SR Research, Mississauga, ON, Canada) was used to record eye movements at $1 \mathrm{KHz}$. Saccade initiation was measured using the algorithm provided by SR Research. This algorithm uses a saccadic velocity threshold of $30 \%$, a saccade acceleration threshold $8000^{\circ} / \mathrm{s}^{2}$ and a saccade motion threshold of $0.15^{\circ}$. Stimulus display and oculomotor data collection were synchronized on a frame-by-frame basis using Experimental Builder (SR Research, Mississauga, ON, Canada). Figure 1A depicts the time line of stimuli presentation on the screen facing the subject. Each trial started with an initial fixation period of a small empty box $\left(1.4 \times 1.4^{\circ}\right)$ appearing on the screen for a random duration $(850 \pm 100 \mathrm{~ms}$; Figure 1A). At the end of this period four additional empty square "boxes" appeared on the screen at an eccentricity of $8^{\circ}$ together with a warning stimulus $S_{1}$ that was briefly presented in the central box for $50 \mathrm{~ms}$. Extinction
TABLE 1 | Summary of drug treatments received by patients.

\begin{tabular}{|c|c|c|c|c|c|c|}
\hline Patient & 1 & 2 & 3 & 4 & 5 & 6 \\
\hline p01 & SNRI & Non-BDZ & BDZ & ATA & $\mathrm{BDZ}$ & \\
\hline p03 & MRA & Non-BDZ & & & & \\
\hline p04 & SSRI & $\mathrm{BDZ}$ & & & & \\
\hline p05 & BDZ & Non-BDZ & $\mathrm{NDRI}$ & & & \\
\hline p06 & NIL & & & & & \\
\hline $\mathrm{p} 07$ & ATA & SSRI & & & & \\
\hline p08 & $\mathrm{NDRI}$ & & & & & \\
\hline p09 & MRA & & & & & \\
\hline p11 & SSRI & ATA & & & & \\
\hline p12 & SSRI & ATA & NDRI & & & \\
\hline p13 & SNRI & ATA & & & & \\
\hline p14 & SSRI & & & & & \\
\hline p15 & SNRI & Non-BDZ & BDZ & & & \\
\hline p17 & NIL & & & & & \\
\hline p18 & SSRI & & & & & \\
\hline p19 & NIL & & & & & \\
\hline p20 & NIL & & & & & \\
\hline $\mathrm{p} 21$ & SNRI & Non-BDZ & Non-BDZ & SARI & ATA & ATA \\
\hline p25 & SSRI & & & & & \\
\hline p26 & SSRI & $\mathrm{BDZ}$ & BDZ & & & \\
\hline p28 & SNRI & ATA & BDZ & BDZ & SARI & $\mathrm{BDZ}$ \\
\hline p29 & SSRI & BDZ & ATA & & & \\
\hline p30 & Non-BDZ & BDZ & & & & \\
\hline p31 & NDRI & & & & & \\
\hline p32 & NIL & & & & & \\
\hline p34 & NIL & & & & & \\
\hline p35 & SNRI & BDZ & Non-BDZ & ATA & & \\
\hline p37 & SNRI & ATA & & & & \\
\hline $\mathrm{p} 41$ & NIL & & & & & \\
\hline
\end{tabular}

Each line represents one patient. Columns show treatments received by each patient (1-6). ATA, atypical antipsychotic; BDZ, benzodiazepine; SNRI, serotoninnorepinephrine reuptake inhibitor; MRA, melatonin receptor agonist; NDRl, norepinephrine-dopamine reuptake inhibitor; NIL, no pharmacological treatment; Non-BDZ, non-benzodiazepine hypnotic; SARI, serotonin antagonist and reuptake inhibitor; SSRI, selective serotonin reuptake inhibitor.

of the S1 stimulus indicated to subjects the beginning of the foreperiod (FP). Subjects were required to fixate on the central box until a target was briefly and randomly presented for $50 \mathrm{~ms}$, in one of the four eccentric boxes (imperative stimulus $S_{2}$ ). The background of the screen was always black and stimuli were white (boxes) or green $\left(S_{1}\right.$ and $S_{2}$ stimuli). One of four different FP durations (400, 900, 1400, and $1900 \mathrm{~ms}$ ) was chosen randomly, each with the same probability (Probability $=0.25$ ). Subjects were required to wait until targets appeared in the eccentric box before making a saccade (black arrowhead on Figure 1A). Saccadic latency (reaction time) was defined as the time elapsed between the appearance of the eccentric target and movement onset. Saccades that occurred during the foreperiod (period indicated with a red line on Figure 1A) are here referred to as "premature saccades" (red traces on Figure 1B). The propensity to initiate premature saccades will be referred to as "oculomotor impulsivity." Saccades that occurred after the appearance of the eccentric target (period indicated with a blue line on Figure 1A) are here referred to as "visually guided saccades" (blue traces 


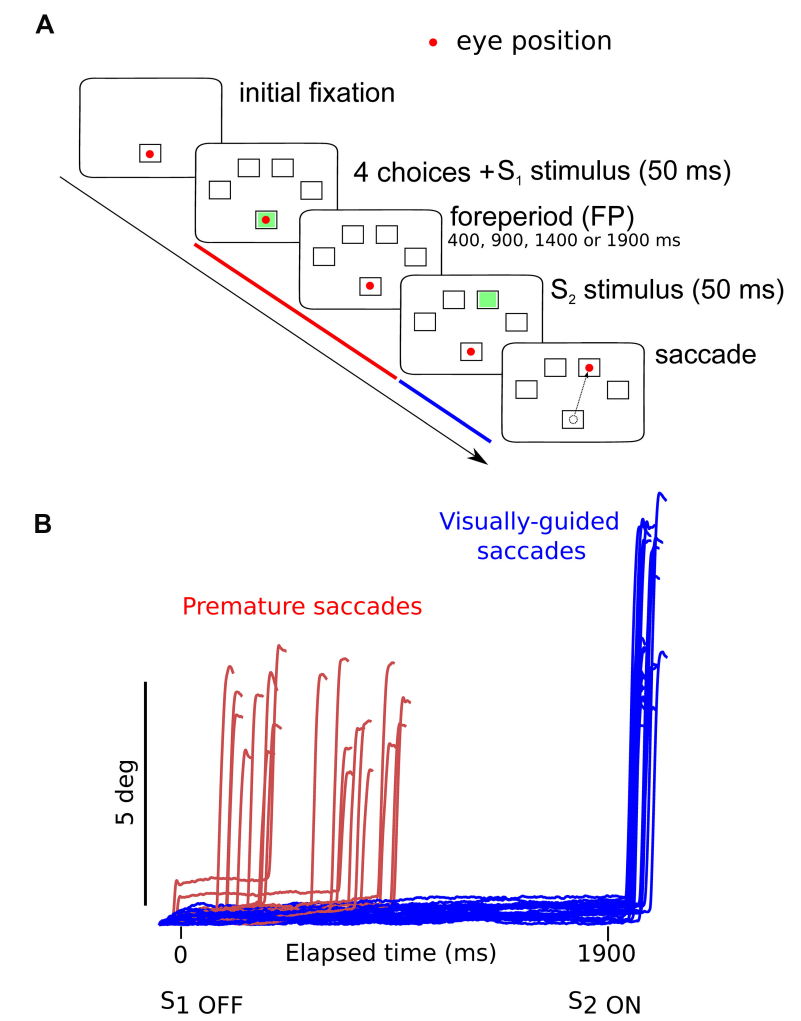

FIGURE 1 | (A) Oculomotor version of the 4-CSRTT task. Schematic representation of the visual display in front of subjects. See text for details. (B) Example of premature (red traces) and visually guided (b/ue traces) saccades during a 1900-ms foreperiod in a control subject (subject \#9). Time zero on the $X$-axis indicates extinction of the warning stimulus $\left(\mathrm{S}_{1} \mathrm{OFF}\right)$. At the end of the FP, the imperative stimulus appeared $\left(S_{2} \mathrm{ON}\right)$. Note that most premature saccades tended to cluster during the early part of the FP. The period during which premature saccades were recorded is represented in red in panel (A). The period during which visually guided saccades were recorded is represented in blue in panel (A)
A linear mixed model approach (LMM) was used to analyze eye movement data. In all analyses, subject identity was used as a random factor to account for the influence of uncontrolled, between-subject variability. To compare the percentage of premature responses, a single average value was computed for each subject before applying the LMM analysis and testing for fixed effects. In order to analyze saccadic latencies, we used a repeated-measures LMM. In this analysis, each saccadic latency measured for each subject was a data point. Indeed, the LMM approach does not require a preliminary averaging of data for each subject and condition. Therefore, it avoids information loss due to data averaging (see West et al., 2015; Boisgontier and Cheval, 2016) and is more selective to test experimental effects and interactions (Baayen et al., 2008). The LMM method is also more robust to normality violations and missing data (e.g., no saccade triggered) than standard ANOVA or ANCOVA.

In summary, LMM provides unbiased analysis of balanced and unbalanced repeated-measures data, detection of withinsubject effects (fixed effects), and individual subject effects (random effects), thereby making the best use of all available data (Laird and Ware, 1982).

The number of degrees of freedom (df) was estimated using the Satterthwaite algorithm calculated by the MIXED algorithm in SPSS 25 (SPSS Inc., Chicago, IL, United States). With this algorithm, the number of $\mathrm{df}$ could be fractional. Significance of observed effects was tested using the F-statistics. The significance threshold $\alpha$ for all analysis was 0.05 .

Results are presented as mean \pm standard error of the mean, unless otherwise specified. Because saccadic reaction time distributions are often non-normal, we used a logarithmic transform of saccadic RT for statistics. For presentation purposes, however, untransformed saccadic latencies in milliseconds are presented on figures.

A preliminary version of this paper was posted on bioRxiv by Hsu et al. (2019).

\section{RESULTS}

\section{Demographics and Clinical Characteristics}

Average age of control subjects was $37.7 \pm 2.4(n=29)$ and $38.4 \pm 2.5(n=29)$ for patients. Age did not differ between groups $(F[1,56]=0.048 ; p=0.827 ; n=29)$. Average scores of the Beck's Depression Inventory-II were $7.6 \pm 1.4$ in controls $(n=25 / 29 ; 4$ untested controls) and $30.1 \pm 13.4$ in patients $(n=22 / 29$; seven untested patients). These group scores were statistically different $\left(\chi^{2}=20.455 ; p<0.001\right)$ confirming the diagnosis of MDD. Average total scores of the BIS-11 were $64.6 \pm 1.2$ in controls $(n=29 / 29)$ and $71.3 \pm 2.0$ in patients $(n=27 / 29)$. These betweengroup scores differed significantly, $\left(\chi^{2}=4.577 ; p=0.032\right)$ with patients scoring higher than controls.

\section{Premature Saccades}

In the oculomotor version of the 4-CSRTT task, subjects were instructed to keep looking at the $S_{1}$ stimulus in the central 


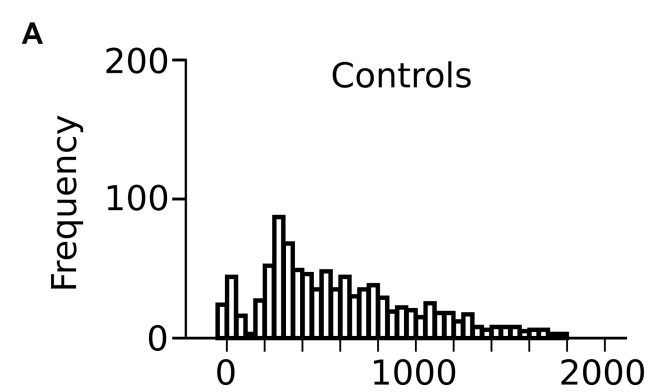

B

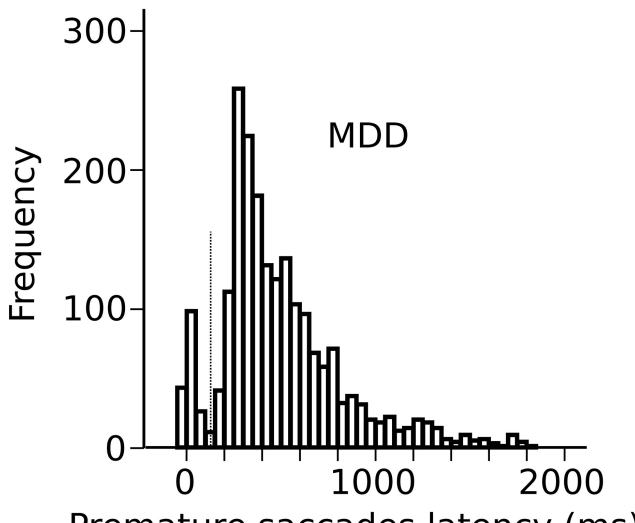

Premature saccades latency (ms)

FIGURE 2 | (A) Latency distribution of all premature saccades in controls pooled together. (B) Latency distribution of all premature saccades in MDD patients. Time zero on the $X$-axis represents disappearance of the $S_{1}$ stimulus.

fixation box and wait for the appearance of the eccentric $S_{2}$ stimulus before initiating a visually guided saccade. However, saccades were often initiated before the appearance of the $S_{2}$ stimulus (see red traces on Figure 1B). These movements will be referred to as "premature" saccades. Figure 2 shows the latency distribution of premature saccades for all controls (Figure 2A) and patients (Figure 2B). Most premature saccades occurred approximately $200 \mathrm{~ms}$ after the offset of the warning stimulus. The percentage of premature saccades was measured and served as an index of inhibition. We found that the percentage of premature saccades was more than twice as high in patients than in controls (in controls: $14 \pm 2 \%, n=29$ subjects, 937 premature saccades/6620 trials; in patients: $31 \pm 5 \%, 2064$ premature saccades/6776 trials) and there was a significant main effect of subject group on this percentage $(F[1,56]=8.4$; $p=0.005)$. Premature saccade latency was longer in controls $(598 \pm 13 \mathrm{~ms} ; n=937)$ than in MDD patients (491 $\pm 7 \mathrm{~ms}$; $n=2066 ; F[1,2926]=13.410 ; p<0.001$ ) supporting the hypothesis of increased oculomotor impulsivity in patients. Next, we investigated whether there was a correlation between trait impulsivity as estimated with the BIS-11 questionnaire and oculomotor impulsivity. However, we found no correlation between the percentage of premature saccades and the BIS-11 score (in MDD patients: $F[1,20]=0.34 ; p=0.856 ; n=22$; seven patients not tested; in controls: $F[1,23]=0.216 ; p=0.647 ; n=25$; four subjects not tested) suggesting that these measures reflect different facets of impulsivity.

\section{Influence of Temporal Preparation on Visually Guided Saccades}

Most saccades were visually guided, as expected, in accord with the instructions provided to subjects (blue traces on Figure 1B; $76 \%$ visually guided saccades, $9451 / 12452$ saccades all subjects included; average latency $239 \pm 1 \mathrm{~ms}, n=5377$ saccades; MDD: $259 \pm 1 \mathrm{~ms}, n=4074$ saccades). Figure 3A shows the relationship between saccadic latency and foreperiod duration in the two groups of subjects. This relationship will be referred to as the RT-FP function. It can be observed that saccadic latencies were similar between groups, if current FP duration was $400 \mathrm{~ms}$. But there was an increasing difference between groups for FP duration longer than $400 \mathrm{~ms}$. We found a statistically significant main effect of FP duration $(F[3,9394.324]=274.374 ; p<0.001)$ on saccadic latency, but no significant main effect of group on average saccadic latency $(F[1,55.082]=0.472 ; p=0.495)$. The interaction between FP duration and subject group was significant $(F[3,9394.324]=22.715 ; p<0.001)$. These results show that average reaction time was statistically similar in controls and MDD subjects but that temporal preparation was reduced in the latter group.

In order to better understand the origin of the different RT-FP functions between groups, we analyzed the influence of previous $\mathrm{FP}$ duration $\left(\mathrm{FP}_{\mathrm{n}-1}\right)$ on saccadic latency during the current FP. For instance, if current FP duration was $400 \mathrm{~ms}$ and $\mathrm{FP}_{\mathrm{n}-1}$ was $400 \mathrm{~ms}$ as well, saccadic latency could be shorter than if the same FP was preceded by $\mathrm{FP}_{\mathrm{n}-1}$ of 1900 ms (see Figure 3B). Statistically, we found that there was a significant main effect of $\mathrm{FP}_{\mathrm{n}-1}$ duration $(F[3,9314.030]=22.841 ; p<0.001)$ on saccadic latency, but not subject group $(F[1,55.229]=1.077 ; p=0.304)$. The interaction between $\mathrm{FP}_{\mathrm{n}-1}$ duration and subject group was not significant $(F[3,9314.030]=1.633 ; p=0.179)$. These results suggest that the $\mathrm{FP}_{\mathrm{n}-1}$ effect was present and similar in both controls and patients. Therefore, the reduced FP effect that we found could not be due to a reduced influence of $\mathrm{FP}_{\mathrm{n}-1}$ duration held in short-term memory. This conclusion was reinforced by the analysis presented on Figure $3 \mathrm{C}$ that shows the influence of $\mathrm{FP}_{\mathrm{n}-1}$ as a function of FP duration in controls and MDD patients. The influence of $\mathrm{FP}_{\mathrm{n}-1}$ was stronger for short $\mathrm{FP}$ durations than longer ones. This effect is often referred to as the "asymmetry" of the sequential effect (Los et al., 2017). This asymmetry was present in both groups and was statistically similar (no significant interaction between $\mathrm{FP}, \mathrm{FP}_{\mathrm{n}-1}$ and subject group: $F[9,9290.307]=1.38 ; p=0.190]$ ). However, more than one step back into the past could still play a significant role in the timing of eye movements and explain the different RT-FP functions between groups. Therefore, we investigated whether $\mathrm{FP}_{\mathrm{n}-1}, \mathrm{FP}_{\mathrm{n}-2}, \mathrm{FP}_{\mathrm{n}-3}, \mathrm{FP}_{\mathrm{n}-4}$ could also play a significant role in determining saccadic latency during the current $\mathrm{FP}_{\mathrm{n}}$ in both groups. We found that $\mathrm{FP}_{\mathrm{n}-1}, \mathrm{FP}_{\mathrm{n}-2}, \mathrm{FP}_{\mathrm{n}-3}$, and $\mathrm{FP}_{\mathrm{n}-4}$ played a significant role in determining saccadic latency in controls $\left(\mathrm{FP}_{\mathrm{n}-1}, F[3,4935.335]=16.090 ; p<0.001 ; \mathrm{FP}_{\mathrm{n}-2}\right.$, $F[3,4935.316]=9.382 ; p<0.001 ; \mathrm{FP}_{\mathrm{n}-3}, F[3,4935.283]=4.126$; 
A

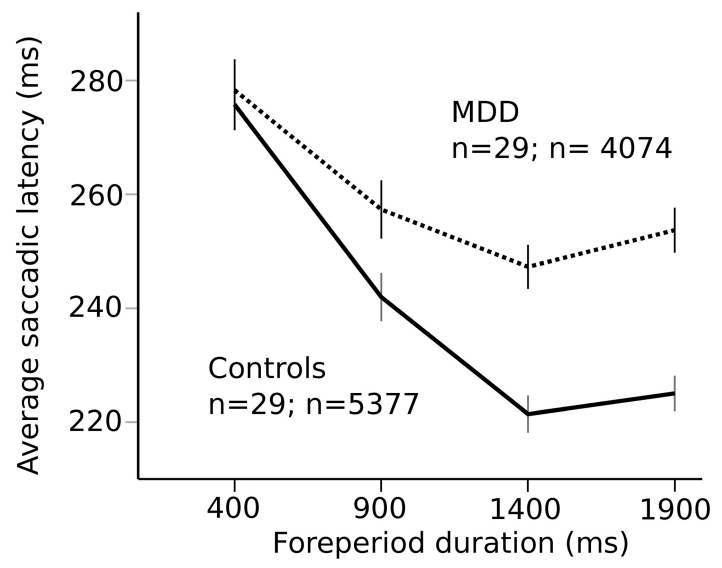

C

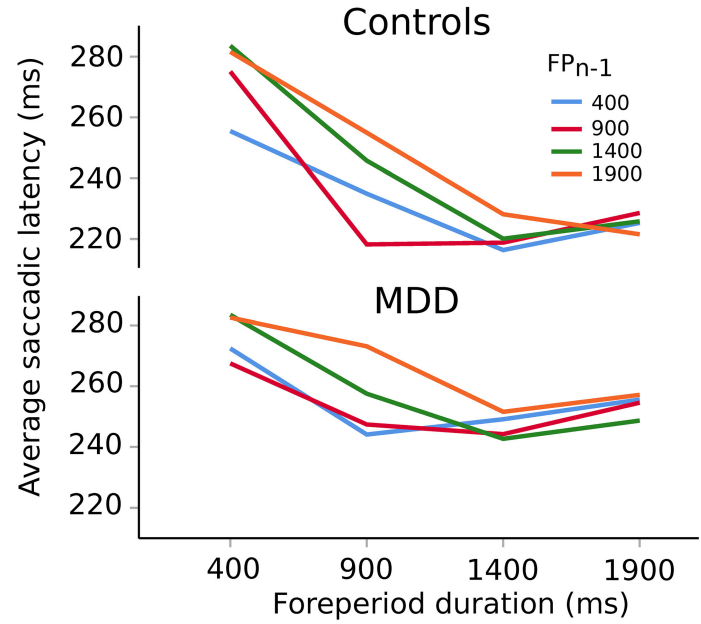

B

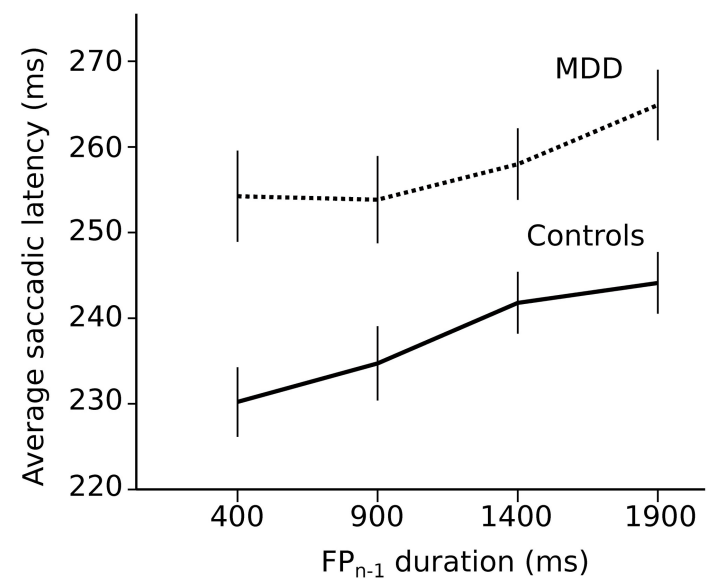

D

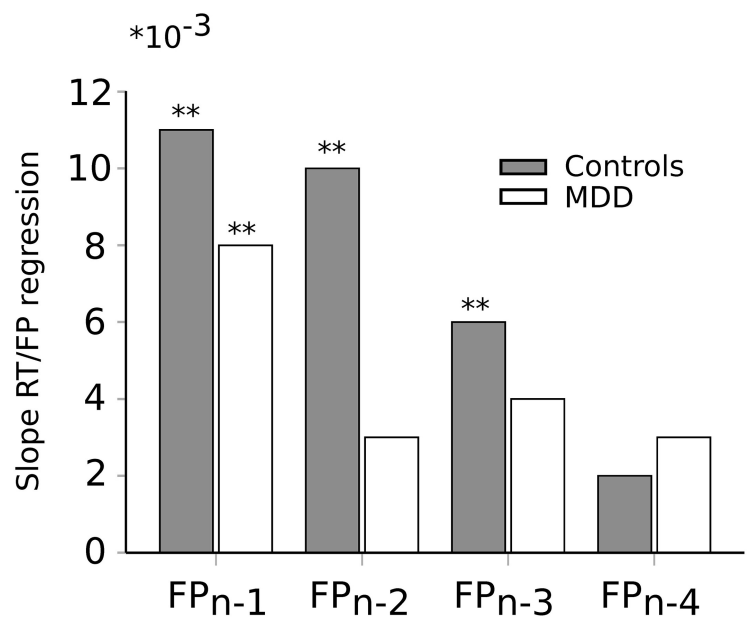

FIGURE 3 | (A) RT-FP function in controls (continuous line; $n=29$ subjects; $n=5377$ saccades) and MDD patients (dotted line; $n=29$ subjects; $n=4074$ saccades). Average values of saccadic latency (in milliseconds) and 95\% confidence interval. Note the shallower slope of the function between 400 and 1400 ms FP durations. (B) Relationship between previous foreperiod duration (FP $\mathrm{n}_{-1}$ ) and saccadic latency in controls (continuous line) and MDD patients (dotted line). Same data set as on panel (A). (C) Asymmetry of the foreperiod effect. Colors shows the duration of the previous FP (FP $n-1)$. Same data as in panel (A) but with a classification of saccadic latency according to previous FP. The influence of $F P_{n-1}$ is larger for short FP durations. (D) Graphical representation of the slope of the linear relationships between previous FPs and saccadic latency for an increasing number of trials back into the past (1-4) in controls (dark bars) and MDD patients (open bars). Same data set as on panel (A). The symbol * is used to represent multiplication; ** indicates $P<0.01$.

$\left.p=0.006 ; \mathrm{FP}_{\mathrm{n}-4}, F[3,4935.237]=3.562 ; p=0.014\right)$. But only $\mathrm{FP}_{\mathrm{n}-1}$ played a significant role on saccadic latency in MDD patients $\left(\mathrm{FP}_{\mathrm{n}-1}, F[3,3654.868]=6.270 ; p<0.001\right)$. Therefore, the reduced FP effect found in patients could be attributed to a different processing of past FP durations, with a faster decay of the memory trace of previous FPs in MDD patients. This is further illustrated on Figure 3D, which shows the value of the slope of a multiple linear regression analysis between previous FPs and saccadic latency. Star symbols indicate when slopes were significantly different from zero. This figure shows a faster extinction of the influence of previous FPs on movement latency in MDD patients than in controls.

Analyses presented above suggest that short-term temporal memory of previous FPs affected saccadic latency in MDD patients differently than in controls. Temporal preparation, however, could also be guided by a sense of elapsed time during the current FP. Therefore, we applied a hierarchical linear regression analysis in both groups using two different models. In controls, the first model contained $\mathrm{FP}_{\mathrm{n}-1}, \mathrm{FP}_{\mathrm{n}-2}$, $\mathrm{FP}_{\mathrm{n}-3}$, and $\mathrm{FP}_{\mathrm{n}-4}$ as independent factors. The second model contained $\mathrm{FP}_{\mathrm{n}-1}, \mathrm{FP}_{\mathrm{n}-2}, \mathrm{FP}_{\mathrm{n}-3}, \mathrm{FP}_{\mathrm{n}-4}$, and $\mathrm{FP}_{\mathrm{n}}$ as independent factors and will be referred to as "model 2." The aim was to determine the contribution of both short-term temporal memory $\left(\mathrm{FP}_{\mathrm{n}-x}\right)$ and current foreperiod $\left(\mathrm{FP}_{\mathrm{n}}\right)$ in movement latency. In controls, we found that both model $1(F[4,5213]=17.269$; $p<0.001)$ and model $2(F[5,5212]=89.276 ; p<0.001)$ provided a significant explanation of observed variance. The coefficient of determination for model 1 was $r^{2}=0.013$ 
$(p<0.001)$; however, the coefficient of determination for model 2 was higher with $r^{2}=0.079$. Therefore, $r^{2}$ variation due to adding current $\mathrm{FP}_{\mathrm{n}}$ in the model was approximately $7 \%(0.066)$ and the $F$-value variation related to this addition was significant $(F[1,5212]=372.384 ; p<0.001)$. In patients, we found that both model $1(F[1,4038]=12.559 ; p<0.001)$ and model $2(F[2,4037]=38.633 ; p<0.001)$ also provided a significant explanation of the variance observed. The coefficient of determination for model 1 was weak $r^{2}=0.003$ but significant $(p<0.001)$. But the coefficient of determination for model 2 was higher with $r^{2}=0.018$ and the $r^{2}$ variation due to adding $\mathrm{FP}_{\mathrm{n}}$ in the model was approximately $2 \%(0.016)$. F-value variation related to the addition of $\mathrm{FP}_{\mathrm{n}}$ in the model was significant $(F[1,4037]=64.511 ; p<0.001)$. In summary, the FP effect was observed in both groups but in MDD patients memory of previous FPs declined faster, given that only $\mathrm{FP}_{\underline{n}-1}$ played a significant role on saccadic latency. Using a hierarchical model analysis, we showed that the model including previous FPs and current $\mathrm{FP}_{\mathrm{n}}$ explained a larger proportion of the variance of saccadic latency. Influence of the current FP was present in both controls and patients but its influence was weaker in the latter group. Therefore, the reduced influence of current FP on saccadic latency co-occurred with a reduced short-term temporal memory in MDD patients.

\section{Was Short-Term Memory in the Spatial Domain Similarly Affected in MDD Patients?}

If the answer to this question were positive, then observed effects were not specific to temporal preparation. Instead, they might reflect a more general short-term memory impairment in patients. In order to determine whether there was a significant effect of previous target position, we compared saccadic latency when the target appeared at the same or at a different spatial location during the previous trial. We hypothesized that there could be a response facilitation by repetition in the temporal domain only, but not in the spatial domain. Indeed, we found no evidence of a significant interaction effect between subject group and previous target location on saccadic latency $(F[1,9318.688]=0.081 ; p=0.776$; see Figure 4A). We applied the same approach to temporal preparation by comparing saccadic reaction time when $\mathrm{FP}_{\mathrm{n}}$ and $\mathrm{FP}_{\mathrm{n}-1}$ were the same or different. In the temporal domain, there was a significant interaction between subject group and FP duration on saccadic latency (same or different; $F[1,8907.674]=4.077 ; p=0.043$; see Figure 4B). These results show that temporal short-term memory was selectively affected in patients, but the same was not true for spatial shortterm memory of target location.

\section{Influence of Oculomotor Impulsivity on Temporal Preparation}

As hypothesized in the Introduction section, the shape of the RT-FP function could be influenced by oculomotor impulsivity. Therefore, we compared the RT-FP functions in low and high oculomotor impulsivity patients and controls. Patients were categorized into two groups, using the median of the percentage
A

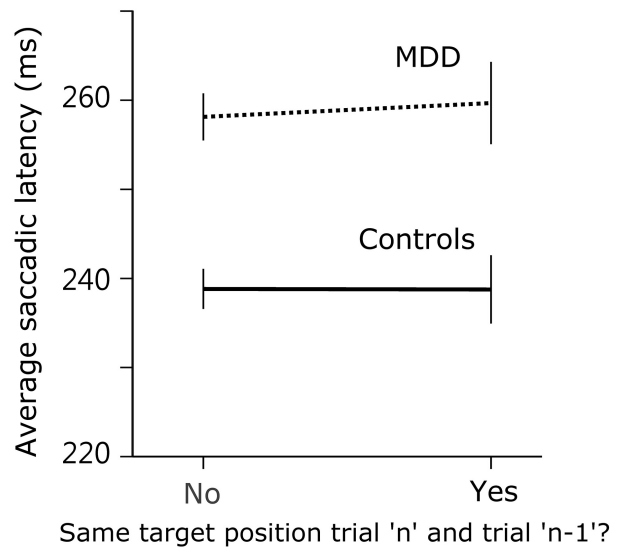

B

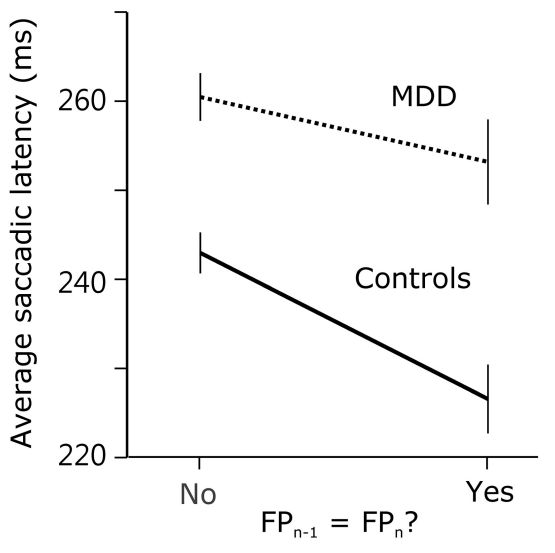

FIGURE 4 | Comparison of spatial and temporal short-term temporal memory. (A) Saccadic latency in MDD and control subjects when the visual target was at the same position during trial ${ }_{n}$ as during trial ${ }_{n-1}$, or not. (B) Saccadic latency when FP duration was the same during trial $\mathrm{n}_{n}$ as during trial $\mathrm{l}_{n-1}$, or not.

of premature saccades distribution in this group (median $=21 \%$ ). The low impulsivity MDD group produced less than 21\% premature responses ( $n=15$ subjects; $n=2864$ saccades); the high impulsivity MDD group, more than $21 \%$ ( $n=14$ subjects; $n=1210$ saccades). We re-examined the FP effect within these two groups. Figure 5A shows that the asymmetric FP effect was present in the low impulsivity group, but it was considerably altered in the high impulsivity group. Accordingly, a significant interaction between impulsivity group and FP duration on saccadic latency was found $(F[3,4048.361]=4.655 ; p=0.003)$. In high oculomotor impulsivity patients, saccadic latencies for the $400 \mathrm{~ms}$ duration FP were reduced by approximately $30 \mathrm{~ms}$. Figure 5B shows the same analysis applied to control subjects (median percentage of premature saccades: median $=10 \%$ ). The RT-FP function in high ( $n=15$ subjects; $n=2313$ saccades) and low impulsivity ( $n=14$ subjects; $n=3064$ saccades) control subjects also significantly differed (significant interaction between impulsivity group and FP duration on saccadic latency; $F[3,5342.181]=4.125 ; p=0.006)$, but to a lesser extent. Indeed, as already mentioned, oculomotor impulsivity was lower in this 
A

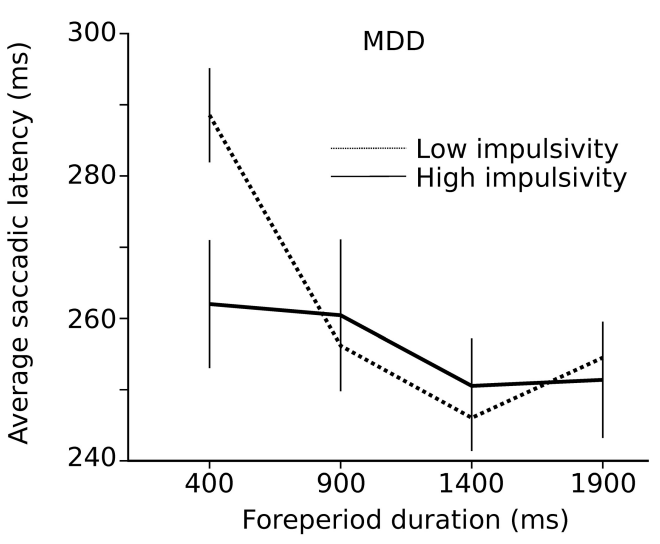

C

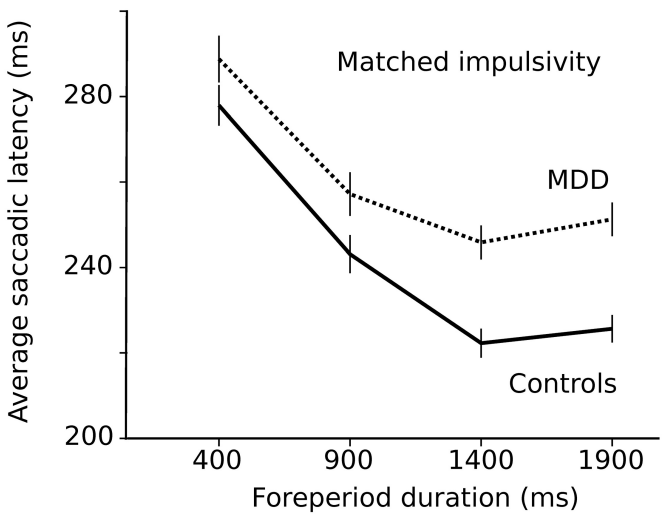

B

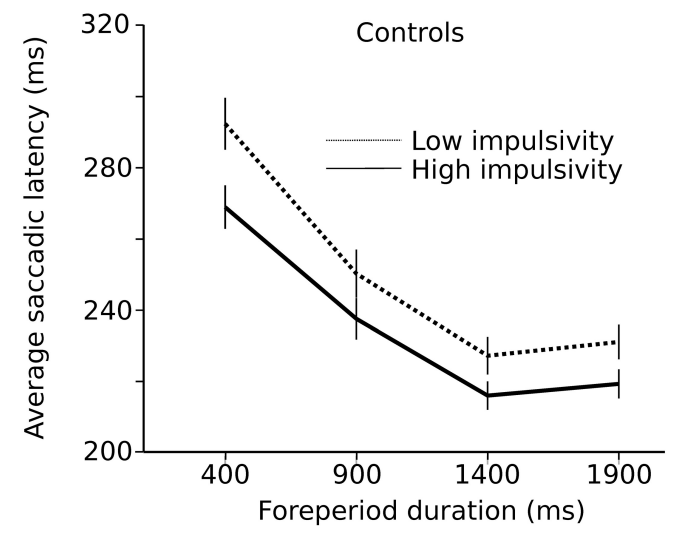

D

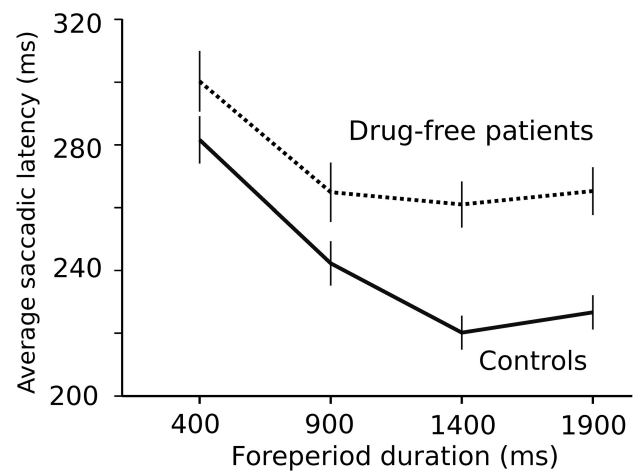

FIGURE 5 | (A) Influence of impulsivity on the RT-FP function in MDD patients (low impulsivity; $n=15$ subjects; $n=2864$ saccades; high impulsivity group: $n=14$ subjects, $n=1210$ saccades). (B) Same relationship in controls (low impulsivity; $n=15$ subjects; $n=2864$ saccades; high impulsivity group: $n=14$ subjects, $n=1210$ saccades). (C) RT-FP function for patients ( $n=19$, nine subjects removed; $n=3546$ saccades) and controls ( $n=26$, two subjects removed; $n=4972$ saccades) that were matched for oculomotor impulsivity. (D) RT-FP function in seven untreated patients ( $n=1305$ saccades) and 7 age-matched controls ( $n=1530$ saccades).

group; therefore, its influence on the RT-FP relationship was less. In summary, impulsivity increased temporal preparation to the temporally proximal target in both controls and patients, with a more pronounced effect in the latter group. It could be hypothesized that the shallower RT-FP relationship in impulsive patients could be partly attributed to a reduced asymmetry of the sequential short-term memory effect. However, we found no interaction between $\mathrm{FP}_{\mathrm{n}}, \mathrm{FP}_{\mathrm{n}-1}$ and subject group $(F[9,4008]=0.691 ; p=0.717$; see Supplementary Figure 1).

The perception of elapsed time, however, could also be modified by depression, independent of impulsivity. In order to test this hypothesis, patients and controls were matched for oculomotor impulsivity. If the shape of the RT-FP relationship was different for the same oculomotor impulsivity, then depression per se is affecting temporal preparation. In order to obtain matched oculomotor impulsivity groups, we removed patients and subjects producing more than $30 \%$ premature responses and identified two overlapping groups - controls ( $n=26,2$ subjects removed; $n=4972$ saccades) and depressed patients ( $n=19,9$ subjects removed; $n=3546$ saccades) producing 12 and $14 \%$ of premature responses, respectively. Figure 5C shows that the RT-FP functions were different, nevertheless, in patients and controls (significant interaction; $F[3,8467.292]=13.082 ; p<0.001)$ with a reduced $\mathrm{FP}$ effect in patients. Therefore, the shape of the RT-FP function could be altered by both oculomotor impulsivity and depression.

One additional factor that could influence temporal preparation is drug treatments administered to MDD patients. Most subjects (22/29) received several drug treatments that could have influenced temporal preparation (see Table 1). Due to ethical considerations, it would not have been acceptable to ask patients to suspend their treatment for research purposes. But seven subjects were investigated before the start of the treatment and temporal preparation could be estimated $(n=1305$ saccades). Figure 5D shows a strong alteration of the RT-FP function for drug-free patients, compared to age-matched controls $(n=1530$ saccades). This subset of seven, untreated subjects presented a similar alteration of the RT-FP function as was found when all subjects were pooled together (see Figure 3A), and a significant interaction between group and FP 
duration on saccade latency was found $(F[3,2815.141]=10.639$; $p<0.001)$. This result suggests that temporal preparation alteration in depression is a robust observation, and that it is not caused by drug treatment. However, we found no correlation between the BDI score and the slope of the RT-FP relationship (Pearson correlation, $-0.032, p=0.88, n=22$ ).

\section{Influence of Trait Impulsivity on Temporal Preparation}

In the present study, the percentage of premature responses was used to evaluate oculomotor impulsivity. The percentage of premature responses was not correlated with the BIS-11 score (see above). However, could the BIS-11 score predict the shape of the RT-FP function? In order to answer this question, we divided MDD patients into a high and low impulsivity groups based on the median of the total BIS-11 score. The same LMM procedure was applied to test whether the RTFP function was different between groups. However, we found that there was no main effect of trait impulsivity on saccadic RT $(F[1,24.250]=0.001 ; p=0.973)$. Moreover, no significant interaction was found between trait impulsivity and FP duration $(F[3,3777.549]=2.235 ; p=0.082)$. In conclusion, temporal preparation was not significantly altered by trait impulsivity.

\section{DISCUSSION}

The aim of this study was to determine how temporal preparation and short-term temporal memory were affected in MDD as well as the influence of the lack of inhibitory control (or impulsivity). In general, we found that temporal preparation was reduced in MDD compared with age-matched healthy controls. We suggest that psychomotor retardation could not fully explain observed results. Indeed, our analysis revealed that average reaction time to visual targets was not statistically different between groups. We found that temporal preparation depended on both the duration of the current FP and short-term memory of previous FPs. In MDD, the influence of current FP duration was reduced and the decay of short-term temporal memory was faster. Indeed, the influence of previous FPs was 3-4 trials deep in controls, but only one trial deep in depressed patients. A significant reduction of the FP effect was found in seven MDD patients who were not undergoing pharmacological treatment; this finding suggests that abnormal temporal preparation occurs independently of therapeutic drugs. In addition, we found that lack of inhibition also influenced temporal preparation. The RT-FP function was statistically flat in high oculomotor impulsivity patients.

\section{Premature Saccades and Oculomotor Impulsivity}

Premature saccades reflected the lack of inhibitory control during the foreperiod. Inhibitory control plays a crucial role while waiting for an expected event (Logan et al., 1997) and motor responses occurring before the appearance of the imperative $\mathrm{S}_{2}$ stimulus must be actively suppressed. We observed that premature saccades clustered between 200 and $400 \mathrm{~ms}$ after
$S_{1}$ offset. We suggest that two hypotheses could explain this observation. First, there could be an increased expectancy of $\mathrm{S}_{2}$ appearance after a short FP. This increased expectancy could be caused by a more salient representation of the short FP in memory. Second, response inhibition might be reduced at the beginning of the FP. But the observation that shortterm temporal memory was reduced in MDD patients does not support the first hypothesis. Indeed, a reduced memory span and an increased saliency of memory traces of short FPs are inconsistent. On the other hand, the second hypothesis is supported by observations made in humans and rodents. It has been suggested that the frontal and prefrontal cortices could exert inhibitory control over behavior, cognition and emotions although there is not yet a consensus about the networks involved in the different domains of inhibition (Jahanshahi and Rothwell, 2017). Interestingly, Narayanan et al. (2006) have shown that in rat reversible inactivation of the dorsomedial prefrontal cortex caused an increase in premature responses and an accelerated reaction time after a short foreperiod, just as was observed in the present study. Therefore, premature saccades might reveal a lack of top-down inhibitory control exerted by the prefrontal cortex; this would be particularly important at the beginning of the FP. Later, during the FP, an excitatory drive could prepare movement initiation when the imperative stimulus occurs. The inhibitory control normally opposes this excitatory drive. But if the inhibitory drive were reduced then the excitatory drive could trigger either a premature response during the FP or a short latency saccade to the imperative visual target during a $400 \mathrm{~ms}$ FP. An increased occurrence of premature responses is often observed in psychiatric disorders and addictions where impulsivity is increased (Dalley and Robbins, 2017; Paasche et al., 2018). Among psychiatric diseases, MDD seems to be a special case. It is usually associated with heightened inhibitory control and reduced motor activity (American Psychiatric Association, 2013). But increased impulsivity has also been reported (Corruble et al., 2003) and top-down inhibitory control is likely to be affected by MDD (Palmwood et al., 2017). This hypothetical reduction of top-down inhibitory control in MDD could be related to reduced serotoninergic neurotransmission. Indeed, it has been shown in humans that a dietary tryptophan depletion procedure that reduces serotonin neurotransmission causes waiting impulsivity (Dalley and Roiser, 2012; Worbe et al., 2014). Therefore, increased oculomotor impulsivity could be a consequence of altered serotoninergic transmission in depressed patients (Coppen, 1967; see Yohn et al., 2017). The saccadic system is a high gain system constantly kept under strong inhibitory control in order to avoid unwanted eye movements (Missal and Keller, 2002; Otero-Millan et al., 2018) and this delicate balance could be easily perturbed by reduced top-down inhibitory control (see review in Pouget et al., 2017). Additionally, increased impulsivity could also be related to therapeutic drugs taken by subjects. Indeed, the RT-FP function of untreated subjects was similar to the one observed in low-impulsivity patients in general. Measuring the percentage of premature saccades could be a clinical tool to estimate the influence of pharmacological treatment on impulsive behavior. 
The absence of correlation between the percentage of premature saccades and trait impulsivity (BIS-11 total score) supports the hypothesis that impulsivity is a complex construct with different aspects and several different neurotransmitters involved (Evenden, 1999; Dalley and Robbins, 2017). Premature responses characterize the motor side of impulsivity but does not reflect higher order cognitive aspects of this phenomenon.

\section{The Origin of Temporal Preparation}

Trace conditioning model suggests that the shape of the RT-FP function could be explained by a single learning mechanism based on previously experienced FPs (Los et al., 2014). In this model, inhibition during $\mathrm{FP}_{\mathrm{n}-1}$ causes a longer RT during the current FP. This explains the asymmetry of the FP effect. Therefore, less inhibitory control (oculomotor impulsivity) should be associated with a reduced influence of short-term temporal memory and a shallower slope of the RT-FP function in MDD. Although the slope of the RT-FP function was reduced in impulsive patients, there was still a significant influence of $\mathrm{FP}_{\mathrm{n}-1}$. We could not validate this prediction of the MTP model in our data set. However, an updated model from the same group, the multiple tract theory of temporal preparation (abbreviated as "MTP"; Los et al., 2017) suggested that the shape of the entire RT - FP function reflects accumulated inhibition across all previous trials (stored in memory traces). That is, the complete area below the RT - FP function is an expression of accumulated inhibition. The asymmetry of the sequential effect is a reflection of inhibition according to MTP, where recent memory traces have a higher weight. Our findings also support that the FP effect has multiple origins (Vallesi and Shallice, 2007; Vallesi et al., 2007a,b) both affected in MDD.

When controls and patients were matched for impulsivity, temporal preparation was nevertheless reduced in the latter group. Therefore, impulsivity alone cannot explain observed results and depression by itself reduced temporal preparation. We suggest that depression could reduce the influence of elapsed time on movement latency for long FPs.

Using the exquisite temporal sensitivity of the oculomotor system we have shown that the implicit processing of time is altered in MDD patients. This implicit processing of time probably involves early neuronal activity in the frontal cortex. It has been shown by Los and Heslenfeld (2005) that the effects of previous FPs were paralleled by similar effects on the fronto-central contingent negative variation $(\mathrm{CNV})$. We suggest that this modulation of the CNV by sequence effects (or short-term temporal memory) should be reduced in MDD.

\section{Implicit Timing, Time Perception, and Awareness}

Based on questionnaires, depressed patients report often a "slowing down" of subjective time (Blewett, 1992; Ratcliffe, 2012; see review in Droit-Volet, 2013). This desynchronization between the subjective experience of time and physical time seems to be a characteristic of depressive states without being systematically studied and compared with other cognitive functions. How temporal preparation, explicit timing and awareness of time relate to each other is unknown. Although temporal preparation and time awareness could be considered as very different phenomena they could rest on overlapping neurophysiological mechanisms. We suggest that alteration of these mechanisms could underlie the abnormal temporal organization of thought and behavior often reported in this disease.

\section{DATA AVAILABILITY STATEMENT}

The datasets generated for this study are available on request to the corresponding author.

\section{ETHICS STATEMENT}

The studies involving human participants were reviewed and approved by the Joint Institutional Review Board, Taipei Medical University, Taipei City, Taiwan (N201603080). The patients/participants provided their written informed consent to participate in this study.

\section{AUTHOR CONTRIBUTIONS}

MM and T-YH designed the research and wrote the manuscript. T-YH performed the research. H-CL contributed the unpublished reagents and analytic tools. TL wrote the manuscript.

\section{FUNDING}

This research was supported by the Taiwan Ministry of Science and Technology grants 105-2632-H-038-001-MY3 and 104-2420-H-038-001-MY3 to TL, 104-2410-H-038-012-MY2 and 106-2410-H-038-004-MY2 to T-YH, and the National Fund for Scientific Research (FRS-FNRS, Belgium) grant 31235458 (Research Credit), and an international mobility grant from Wallonie-Bruxelles International (WBI) to MM.

\section{ACKNOWLEDGMENTS}

We would like to express our gratitude to Professor Chi-Hung Juan for sharing equipment that greatly assisted the study. This manuscript has been released as a Pre-Print at bioRxiv.

\section{SUPPLEMENTARY MATERIAL}

The Supplementary Material for this article can be found online at: https://www.frontiersin.org/articles/10.3389/fnbeh.2019. 00258/full\#supplementary-material 


\section{REFERENCES}

Alegria, J., and Delhaye-Rembaux, M. (1975). Sequential effects of foreperiod duration and conditional probability of the signal in a choice reaction time task. Acta Psychol. 39, 321-328. doi: 10.1016/0001-6918(75)90024-4

Ameqrane, I., Pouget, P., Wattiez, N., Carpenter, R. H., and Missal, M. (2014). Implicit and explicit timing in oculomotor control. PLoS One 9:e93958. doi: 10.1371/journal.pone.0093958

American Psychiatric Association, (2013). Diagnostic and Statistical Manual of Mental Disorders, 5th Edn. Arlington, TX: American Psychiatric Publishing, $160-168$.

Baayen, R. H., Davidson, D. J., and Bates, D. M. (2008). Mixed-effects modelling with crossed random effects for subjects and items. J. Mem. Lang. 59, 390-412. doi: 10.1016/j.jml.2007.12.005

Badler, J. B., and Heinen, S. J. (2006). Anticipatory movement timing using prediction and external cues. J. Neurosci. 26, 4519-4525. doi: 10.1523/jneurosci. 3739-05.2006

Barkley, R. A., Edwards, G., Laneri, M., Fletcher, K., and Metevia, L. (2001). Executive functioning, temporal discounting, and sense of time in adolescents with attention deficit hyperactivity disorder (ADHD) and oppositional defiant disorder (ODD). J. Abnorm. Child Psychol. 29, 541-556.

Barnes, G. R. (2008). Cognitive processes involved in smooth pursuit eye movements. Brain Cogn. 68, 309-326. doi: 10.1016/j.bandc.2008.08.020

Beck, A. T., Steer, R. A., and Brown, G. K. (1996). Manual for the Beck Depression Inventory-II. San Antonio, TX: Psychological Corporation.

Blewett, A. E. (1992). Abnormal subjective time experience in depression. Br. J. Psychiatry 161, 195-200. doi: 10.1192/bjp.161.2.195

Boisgontier, M. P., and Cheval, B. (2016). The anova to mixed model transition. Neurosci. Biobehav. Rev. 68, 1004-1005. doi: 10.1016/j.neubiorev.2016.05.034

Bonin-Guillaume, S., Blin, O., and Hasbroucq, T. (2004). An additive factor analysis of the effect of depression on the reaction time of old patients. Acta Psychol. 117, 1-11. doi: 10.1016/j.actpsy.2004.04.002

Burle, B., Tandonnet, C., and Hasbroucq, T. (2010). "Excitatory and inhibitory motor mechanisms of temporal preparation," in Attention and Time, eds A. C. Nobre, and J. T. Coull, (Oxford: Oxford University Press), 243-255.

Burt, D. B., Zembar, M. J., and Niederehe, G. (1995). Depression and memory impairment: a meta-analysis of the association, its pattern, and specificity. Psychol Bull. 117, 285-305. doi: 10.1037//0033-2909.117. 2.285

Collins, C. J., and Barnes, G. R. (2009). Predicting the unpredictable: weighted averaging of past stimulus timing facilitates ocular pursuit of randomly timed stimuli. J. Neurosci. 29, 13302-13314. doi: 10.1523/JNEUROSCI.1636-09.2009

Coppen, A. (1967). The biochemistry of affective disorders. Br. J. Psychiatry 113, 1237-1264.

Correa, A., Triviño, M., Pérez-Dueñas, C., Acosta, A., and Lupiáñez, J. (2010). Temporal preparation, response inhibition and impulsivity. Brain Cogn. 73, 222-228. doi: 10.1016/j.bandc.2010.05.006

Corruble, E., Benyamina, A., Bayle, F., Falissard, B., and Hardy, P. (2003). Understanding impulsivity in severe depression? A psychometrical contribution. Prog. Neuropsychopharmacol. Biol. Psychiatry 27, 829-833. doi: 10.1016/s0278-5846(03)00115-5

Coull, J., and Nobre, A. (2008). Dissociating explicit timing from temporal expectation with fMRI. Curr. Opin. Neurobiol. 18, 137-144. doi: 10.1016/j.conb. 2008.07.011

Coull, J. T., and Droit-Volet, S. (2018). Explicit understanding of duration develops implicitly through action. Trends Cogn. Sci. 22, 923-937. doi: 10.1016/j.tics. 2018.07.011

Dalley, J. W., and Robbins, T. W. (2017). Fractionating impulsivity: neuropsychiatric implications. Nat. Rev. Neurosci. 18, 158-171. doi: 10.1038/nrn.2017.8

Dalley, J. W., and Roiser, J. P. (2012). Dopamine, serotonin and impulsivity. Neuroscience 215, 42-58. doi: 10.1016/j.neuroscience.2012.03.065

Davalos, D., Mioni, G., Grondin, S., and Ortuño, F. (2018). Editorial: time perception and dysfunction: clinical and practical implications. Front. Hum. Neurosci. 12:435. doi: 10.3389/fnhum.2018.00435

de Hemptinne, C., Nozaradan, S., Duvivier, Q., Lefèvre, P., and Missal, M. (2007). How do primates anticipate uncertain future events? J. Neurosci. 27, 4334-4341. doi: 10.1523/jneurosci.0388-07.2007
Degos, B., Ameqrane, I., Rivaud-Péchoux, S., Pouget, P., and Missal, M. (2018). Short-term temporal memory in idiopathic and Parkin-associated Parkinson's disease. Sci. Rep. 8:7637. doi: 10.1038/s41598-018-25751-8

Droit-Volet, S. (2013). Time perception, emotions and mood disorders. J. Physiol. 107, 255-264. doi: 10.1016/j.jphysparis.2013.03.005

Duque, J., Greenhouse, I., Labruna, L., and Ivry, R. B. (2017). Physiological markers of motor inhibition during human behavior. Trends Neurosci. 40, 219-236. doi: 10.1016/j.tins.2017.02.006

Evenden, J. L. (1999). Varieties of impulsivity. Psychopharmacology 146, 348-361. doi: $10.1007 / \mathrm{pl} 00005481$

Gallagher, S. (2012). Time, emotion, and depression. Emot. Rev. 4, 1-6.

Greenhouse, I., Sias, A., Labruna, L., and Ivry, R. B. (2015). Nonspecific inhibition of the motor system during response preparation. J. Neurosci. 35, 10675-10684. doi: 10.1523/JNEUROSCI.1436-15.2015

Hsu, T. Y., Lee, H. C., Lane, T. J., and Missal, M. (2019). Temporal preparation and short-term temporal memory in depression. bioRxiv [Preprint]. doi: 10.1101/ 708370

Jahanshahi, M., and Rothwell, J. C. (2017). Inhibitory dysfunction contributes to some of the motor and non-motor symptoms of movement disorders and psychiatric disorders. Philos. Trans. R. Soc. Lond. B Biol. Sci. 372:20160198. doi: 10.1098/rstb.2016.0198

Janssen, P., and Shadlen, M. N. (2005). A representation of the hazard rate of elapsed time in macaque area LIP. Nat. Neurosci. 8, 234-241. doi: 10.1038/ nn 1386

Laird, N. M., and Ware, J. H. (1982). Random-effects models for longitudinal data. Biometrics 38, 963-974.

Lebon, F., Greenhouse, I., Labruna, L., Vanderschelden, B., Papaxanthis, C., and Ivry, R. B. (2016). Influence of delay period duration on inhibitory processes for response preparation. Cereb. Cortex 26, 2461-2470. doi: 10.1093/cercor/bhv069

Logan, G. D., Schachar, R., and Tannock, R. (1997). Impulsivity and inhibitory control. Psychol. Sci. 8, 60-64. doi: 10.1111/j.1467-9280.1997.tb00 545.x

Los, S. A. (2013). The role of inhibition in temporal preparation: evidence from a go/no-go task. Cognition 129, 328-344. doi: 10.1016/j.cognition.2013.07.013

Los, S. A., and Heslenfeld, D. J. (2005). Intentional and unintentional contributions to nonspecific preparation: electrophysiological evidence. J. Exp. Psychol. Gen. 134, 52-72. doi: 10.1037/0096-3445.134.1.52

Los, S. A., Kruijne, W., and Meeter, M. (2014). Outlines of a multiple trace theory of temporal preparation. Front. Psychol. 5:1058. doi: 10.3389/fpsyg.2014.01058

Los, S. A., Kruijne, W., and Meeter, M. (2017). Hazard versus history: temporal preparation is driven by past experience. J. Exp. Psychol. Hum. Percept. Perform. 43, 78-88. doi: 10.1037/xhp0000279

Los, S. A., and Van den Heuvel, C. E. (2001). Intentional and unintentional contributions to nonspecific preparation during reaction time foreperiods. J. Exp. Psychol. Hum. Percept. Perform. 27, 370-386. doi: 10.1037//0096-1523. 27.2.370

Missal, M., and Keller, E. L. (2002). Common inhibitory mechanism for saccades and smooth-pursuit eye movements. J. Neurophysiol. 88, 1880-1892. doi: 10 . 1152/jn.2002.88.4.1880

Msetfi, R. M., Murphy, R. A., and Kornbrot, D. E. (2012). The effect of mild depression on time discrimination. Q. J. Exp. Psychol. 65, 632-645. doi: 10.1080/ 17470218.2011.608908

Narayanan, N. S., Horst, N. K., and Laubach, M. (2006). Reversible inactivations of rat medial prefrontal cortex impair the ability to wait for a stimulus. Neuroscience. 139, 865-876. doi: 10.1016/j.neuroscience.2005. 11.072

Niemi, P. (1981). Foreperiod and simple reaction time. Psychol. Bull. 89, 133-162. doi: 10.1037//0033-2909.89.1.133

Niemi, P., and Näätänen, R. (1981). Foreperiod and simple reaction time. Psychol. Bull. 89, 133-162. doi: 10.1037//0033-2909.89.1.133

Nobre, A., Correa, A., and Coull, J. (2007). The hazards of time. Curr. Opin. Neurobiol. 17, 465-470.

Nombela, C., Rittman, T., Robbins, T. W., and Rowe, J. B. (2014). Multiple modes of impulsivity in Parkinson's disease. PLoS One 9:e85747. doi: 10.1371/journal. pone. 0085747

Oberfeld, D., Thönes, S., Palayoor, B. J., and Hecht, H. (2014). Depression does not affect time perception and time-to-contact estimation. Front. Psychol. 5:810. doi: $10.3389 /$ fpsyg. 2014.00810 
Oswal, A., Ogden, M., and Carpenter, R. H. (2007). The time course of stimulus expectation in a saccadic decision task. J. Neurophysiol. 97, 2722-2730. doi: 10.1152/jn.01238.2006

Otero-Millan, J., Optican, L. M., Macknik, S. L., and Martinez-Conde, S. (2018). Modeling the triggering of saccades, microsaccades, and saccadic intrusions. Front. Neurol. 28:346. doi: 10.3389/fneur.2018.00346

Paasche, C., Weibel, S., Wittmann, M., and Lalanne, L. (2018). Time perception and impulsivity: a proposed relationship in addictive disorders. Neurosci. Behav. Rev. 106, 182-201. doi: 10.1016/j.neubiorev.2018.12.006

Palmwood, E. N., Krompinger, J. W., and Simons, R. F. (2017). Electrophysiological indicators of inhibitory control deficits in depression. Biol. Psychol. 130, 1-10. doi: 10.1016/j.biopsycho.2017.10.001

Patton, J. H., Stanford, M. S., and Barratt, E. S. (1995). Factor structure of the Barratt impulsiveness scale. J. Clin. Psychol. 51, 768-774. doi: 10.1002/10974679(199511)51:6<768::aid-jclp2270510607>3.0.co;2-1

Pouget, P., Murthy, A., and Stuphorn, V. (2017). Cortical control and performance monitoring of interrupting and redirecting movements. Philos. Trans. R. Soc. Lond. B Biol. Sci. 372:1718. doi: 10.1098/rstb.2016.0201

Ratcliffe, M. (2012). Varieties of temporal experience in depression. J. Med. Philos. 37, 114-138. doi: 10.1093/jmp/jhs010

Rucker, J. C., Ying, S. H., Moore, W., Optican, L. M., Büttner-Ennever, J., Keller, E. L., et al. (2011). Do brainstem omnipause neurons terminate saccades? Ann. N. Y. Acad. Sci. 1233, 48-57. doi: 10.1111/j.1749-6632.2011.06170.x

Sheehan, D. V., Lecrubier, Y., Sheehan, K. H., Amorim, P., Janavs, J., Weiller, E., et al. (1998). The Mini-International Neuropsychiatric Interview (M.I.N.I.): the development and validation of a structured diagnostic psychiatric interview for DSM-IV and ICD-10. J. Clin. Psychiatry 59(Suppl. 20), 22-33.

Smith, A., Taylor, E., Rogers, J. W., Newman, S., and Rubia, K. (2002). Evidence for a pure time perception deficit in children with ADHD. J. Child Psychol. Psychiatry 43, 529-542. doi: 10.1111/1469-7610.00043

Spitzer, R. L., Kroenke, K., Williams, J. B. W., and Löwe, B. (2006). A brief measure for assessing generalized anxiety disorder: the GAD-7. Arch. Intern. Med. 166, 1092-1097.

Sweeney, J. A., Strojwas, M. H., Mann, J. J., and Thase, M. E. (1998). Prefrontal and cerebellar abnormalities in major depression: evidence from oculomotor studies. Biol. Psychiatry 43, 584-594. doi: 10.1016/s0006-3223(97)00485-x

Thönes, S., and Oberfeld, D. (2015). Time perception in depression: a metaanalysis. J. Affect. Disord. 175, 359-372. doi: 10.1016/j.jad.2014.12.057

Trillenberg, P., Verleger, R., Wascher, E., Wauschkuhn, B., and Wessel, K. (2000). CNV and temporal uncertainty with "ageing" and "non-ageing" S1-S2 intervals. Clin. Neurophysiol. 111, 1216-1226. doi: 10.1016/s1388-2457(00) 00274- 1
Vallesi, A., and Shallice, T. (2007). Developmental dissociations of preparation over time: deconstructing the variable foreperiod phenomena. J. Exp. Psychol. Hum. Percept. Perform. 33, 1377-1388. doi: 10.1037/0096-1523.33.6.1377

Vallesi, A., Mussoni, A., Mondani, M., Budai, R., Skrap, M., and Shallice, T. (2007a). The neural basis of temporal preparation: insights from brain tumor patients. Neuropsychologia. 45, 2755-2763. doi: 10.1016/j.neuropsychologia.2007.04.017

Vallesi, A., Shallice, T., and Walsh, V. (2007b). Role of the prefrontal cortex in the foreperiod effect: TMS evidence for dual mechanisms in temporal preparation. Cereb. Cortex. 17, 466-474. doi: 10.1093/cercor/bhj163

Vatakis, A., Balcl, F., Di Luca, M., and Correa, A. (2018). Timing and Time Perception: Procedures, Measures, \& Applications. Amsterdam: Brill.

Vogel, D. H. V., Krämer, K., Schoofs, T., Kupke, C., and Vogeley, K. (2018). Disturbed experience of time in depression-evidence from content analysis. Front. Hum. Neurosci. 12:66. doi: 10.3389/fnhum.2018.00066

Voon, V., Irvine, M. A., Derbyshire, K., Worbe, Y., Lange, I., Abbott, S., et al. (2014). Measuring "waiting" impulsivity in substance addictions and binge eating disorder in a novel analogue of rodent serial reaction time task. Biol. Psychiatry 75, 148-155. doi: 10.1016/j.biopsych.2013.05.013

Voon, V., Mehta, A. R., and Hallett, M. (2011). Impulse control disorders in Parkinson's disease: recent advances. Curr. Opin. Neurol. 24, 324-330. doi: 10.1097/WCO.0b013e3283489687

West, B. T., Welch, K. B., and Galecki, A. T. (2015). Linear Mixed Models: A Practical Guide Using Statistical Software, Second Edn. London: Chapman and Hall.

Woodrow, H. (1914). The measurement of attention. Psychol. Monogr. 5, 1-158.

Worbe, Y., Savulich, G., Voon, V., Fernandez-Egea, E., and Robbins, T. W. (2014). Serotonin depletion induces 'waiting impulsivity' on the human four-choice serial reaction time task: cross-species translational significance. Neuropsychopharmacology 39, 1519-1526. doi: 10.1038/npp.2013.351

Yohn, C. N., Gergues, M. M., and Samuels, B. A. (2017). The role of 5-HT receptors in depression. Mol. Brain 10:28. doi: 10.1186/s13041-017-0306-y

Conflict of Interest: The authors declare that the research was conducted in the absence of any commercial or financial relationships that could be construed as a potential conflict of interest.

Copyright (c) 2019 Hsu, Lee, Lane and Missal. This is an open-access article distributed under the terms of the Creative Commons Attribution License (CC BY). The use, distribution or reproduction in other forums is permitted, provided the original author(s) and the copyright owner(s) are credited and that the original publication in this journal is cited, in accordance with accepted academic practice. No use, distribution or reproduction is permitted which does not comply with these terms. 